\title{
DAILY PROSTATE VOLUME AND POSITION MONITORING USING IMPLANTED GOLD MARKERS AND ON-BOARD IMAGING DURING RADIOTHERAPY
}

\author{
Linda Kašaová ${ }^{1,2}$, Igor Sirák ${ }^{1}$, Jan Jansa ${ }^{1}$, Petr Paluska ${ }^{1}$, Jiří Petera ${ }^{1}$
}

Charles University in Prague, Faculty of Medicine and University Hospital Hradec Králové, Czech Republic: Department of Oncology and Radiotherapy'; University of Defence, Faculty of Military Health Sciences, Hradec Králové, Czech Republic ${ }^{2}$

\begin{abstract}
Summary: Purpose: This study aimed to evaluate prostate volume changes and prostate motions during radiotherapy.Methods: In 2010, twenty-five patients were treated for prostate cancer by external beam radiotherapy with implanted fiducial markers. Coordinates of three gold markers on kilovoltage images were calculated daily. Volume changes in target structure were observed through changes in intermarker distances. Differences in patient position between laser-tattoo alignment and gold marker localization were evaluated. Intrafraction motion was assessed by measuring marker displacement on kilovoltage images acquired before and after fraction delivery. Results: Prostate shrinkage was observed in $60 \%$ of patients. The average shrinkage was $7 \%$ of the prostate's initial volume. Corrections after laser-tattoo alignment remained mostly below $1 \mathrm{~cm}$. The difference between marker centroid position on the actual images and the planning images was $2 \pm 1 \mathrm{~mm}$ on average. The extension of intrafraction movements was $7.6 \pm 0.2 \mathrm{~mm}$ on average. Conclusions: In our retrospective study, the possibility for prostate volume changes during radiotherapy was revealed. Intrafraction movements turned out to be the limiting factor in safety margin reduction.
\end{abstract}

Key words: Prostate radiotherapy; Fiducial markers; Target localization; Volume changes

\section{Introduction}

The curative potential of radical prostate radiotherapy strongly depends on the delivered dose of radiation, standardly above 70 Gy $(1,2)$ With modern conformal techniques, it is suitable to irradiate the prostate with a sufficient safety margin, which should be as small as possible to minimize the risk of organ damage (3). For reliable margin reduction, it is necessary to know several uncertainties entering the process of radiotherapy, especially the movement of the target volume, the variation in anatomical conditions etc. (4-6). Several techniques have been developed to ensure precise evaluation and correction of possible errors in treatment. The most precise measures manage to visualize the treatment volume directly.

At the Department of Oncology and Radiotherapy, Faculty Hospital in Hradec Kralove, three gold fiducial markers are routinely implanted into the prostate before radiotherapy to facilitate the figuration on two orthogonal kilovoltage $(\mathrm{kV})$ images acquired in the treatment position before each fraction. By combining these images, a precise target position reference to Clinac (Varian Medical Systems, Palo Alto, CA, USA) isocenter can be set. The data from daily $\mathrm{kV}$ images are helpful in determining the appropriate safety margin regarding intra and interfraction gland movements.
In the present study we tried to assess differences in patient positioning through laser-tattoo alignment and radiopaque markers. Imaging before and after fraction delivery helped in the exploration of intrafraction prostate movement. Three fiducial markers proved to be a sufficient number to allow for the assessment of prostate volume changes which may occur during radiotherapy or androgen deprivation (7). Our daily marker position monitoring made it possible to estimate such target volume variations.

\section{Patients and Methods}

\section{Patients}

Twenty-five patients with T1c-T3bN0M0 prostate adenocarcinoma treated with radical radiotherapy at our department were retrospectively studied. All patients underwent an implantation of three gold markers under short intravenous general anesthesia before treatment planning. The position of marker implantation was controlled under transrectal ultrasound with a brachytherapeutical grid. The pattern of implantation was as follows: one fiducial marker in the apex of the prostate; two markers in the prostate base on the left and right side each. This pattern assures favorable marker distribution over the entire prostate volume for three dimensional reconstructions. 
Treatment planning transversal CT (Siemens AG, Erlangen, Germany) scan with $3 \mathrm{~mm}$ spacing was performed about one week after the implant procedure due to precluding of bleeding, inflammation, or edema. Rectal filling protocol was used to keep the rectum empty during the CT used for planning and at each treatment session. All patients underwent intensity modulated radiotherapy (IMRT) at a dose of 78 Gy in 39 fractions to the prostate, five times per week. Patient positioning on the treatment couch was performed based on body tattoos and laser alignment at first, and after that two rectangular (gantry $0^{\circ}$ and $270^{\circ}$ ) $\mathrm{kV}$ images (OBI (Varian Medical Systems, Palo Alto, CA, USA)) were acquired every fraction. Patient position was corrected online in accordance with Digitally Reconstructed Radiographs (DRRs). Cartesian coordinates of markers were assessed in every pair of such images and the center of their gravity was calculated retrospectively. Coordinate origin was set in the treatment isocenter.

\section{Evaluation}

At first, the distance of each marker from the center of gravity was calculated and plotted onto the graph, where the $\mathrm{x}$ axis represents the number of fractions and the $\mathrm{y}$ axis the distance. All the marker points were even with the line and their angular coefficients were evaluated (Figure 1). Patients with all three negative and all positive tangents were looked at. Those with all negative tangents were evaluated as exhibiting prostate shrinkage and those with all positive ones as exhibiting prostate expansion. Prostate shrinkage/expansion was considered isotropic. In accordance with the structure volume from the planning system, each prostate was substituted with a sphere model. The new prostate volume after the last fraction was calculated based on the shrinkage/expansion rate and the duration of treatment. The t-test was used to compare prostate volumes before and after treatment.

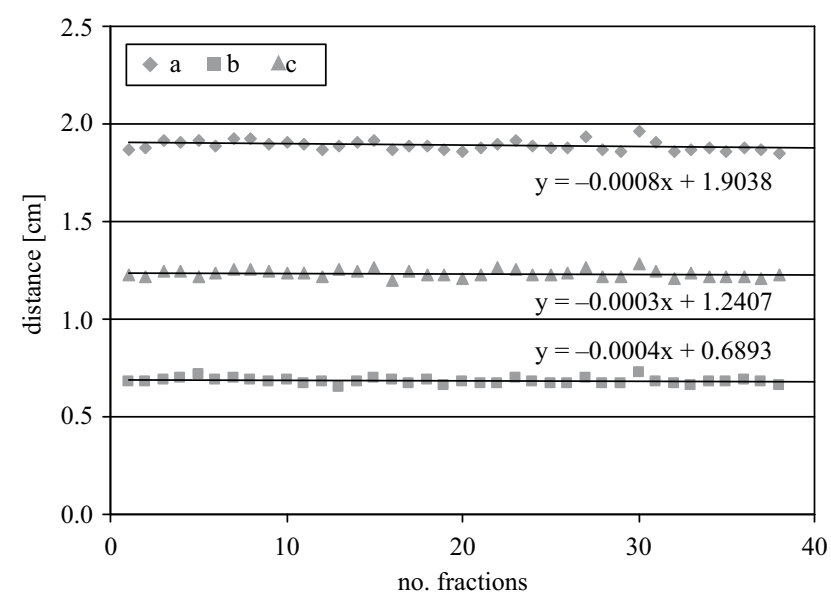

Fig. 1: The distance of each marker (a, b, c) from center of gravity and their angular coefficients for selected patient

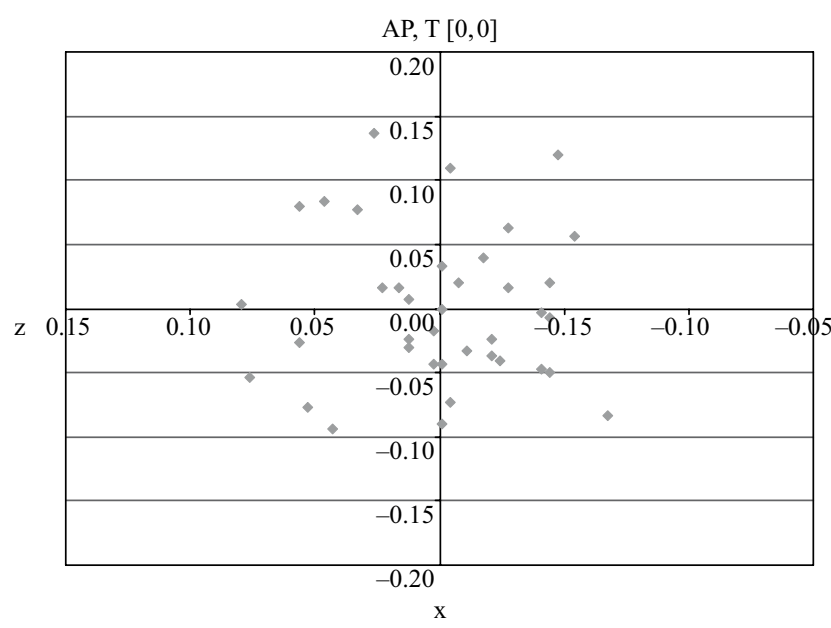

Fig. 2: The positions of markers' centroid in every fraction relative to the reference $([0,0])$ on anterior-posterior images for selected patient in centimeters

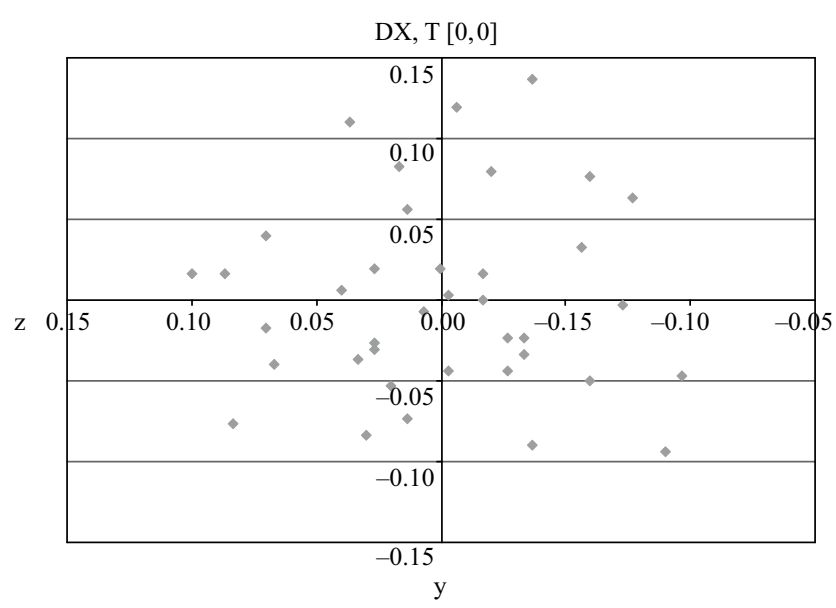

Fig. 3: The positions of markers' centroid in every fraction relative to the reference $([0,0])$ on lateral images for selected patient in centimeters

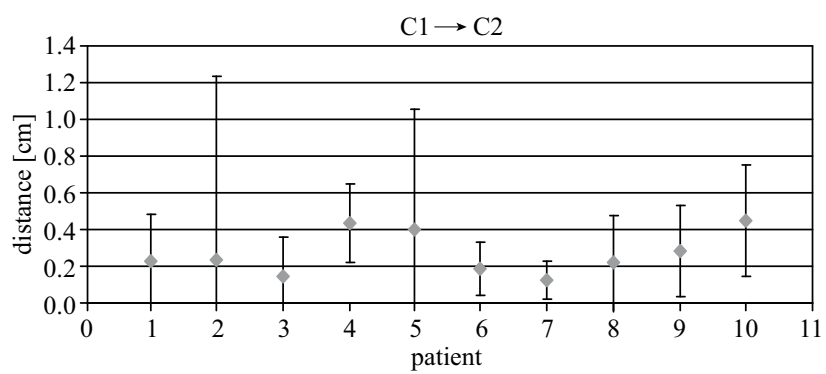

Fig. 4: The average distance of markers' centroid before $(\mathrm{C} 1)$ and after $(\mathrm{C} 2)$ fraction $+2 \sigma$ 
In the second part, the interfractional prostate motion was analyzed. The marker center of mass on the initial CT was set as a reference. The absolute deviation from the marker centroid and its direction were evaluated in every fraction relative to the reference (Figure 2, 3).

Ten patients underwent additional radiographs after daily treatment nine times on average throughout the course of radiotherapy. Intrafractional motion was assessed through differences between the center of gravity coordinates before and after fraction delivery (Figure 4). The rate of displacement was expressed through a sphere, where the marker centroid could be found. The diameter of this sphere was calculated as an average distance between centroids before and after and the sum of their standard deviation.

\section{Results}

All three positive tangents were observed only in two patients. Corresponding prostate expansion for these cases should have been $2.3 \%$ and $3.5 \%$ of the initial volume. On the contrary, all negative tangents in 15 patients $(60 \%)$ were discovered. The shift of every marker to the center of gravity varies between several tenths of a millimeter. The average shrinkage of $7.0 \%$ of the initial volume was calculated (range of variation $2.8-18.6 \%$ ). A statistically significant difference between the initial and final fraction prostate volume was discovered $(p=0.002)$, but no shrinkage rate dependence on the initial volume was recorded.

After initial patient laser-tattoo alignment it was necessary to correct the patient's position in accordance with fiducial markers on OBI images. These corrections remained mostly below 1 centimeter. Even after this procedure, the conformity between the setup and the actual marker centroid was not achieved. The distance between these two points was $2 \pm 1 \mathrm{~mm}$ on average and in $95 \%$ of the instances it was below $\pm 5 \mathrm{~mm}$. No directional dependence was observed.

The mean sphere diameter for intrafraction movement of the markers' center of gravity was $7.6 \pm 0.2 \mathrm{~mm}$ (range of variation $4.8-12 \mathrm{~mm}$ ).

\section{Discussion}

Intraprostatic fiducial marker positioning in patients relies on the premise that the markers reflect the prostate position and do not migrate within or out of the prostate (8). Prostate volume decreases during oncology treatment are well documented $(9,10)$. This phenomenon causes a decrease in intermarker distance and could be distinguished by marker migration (11). Contemporary treatment algorithm expects unchanging prostate volume and shape. In our study it was shown that prostate volume does not need to be equal at the beginning and end of the radiotherapy treatment. Even if observed decrease were not so marked, they could nonetheless lead to an inappropriate shift of critical structures to high dose regions systematically. Although the cohort of patients is too small to provide a definite conclusion, these results correspond with other authors $(12,13)$.

The center of mass of gold seeds was used as an instrument to evaluate prostate setup errors. This approach makes it possible to avoid lapses due to marker displacement. Isocenter position conformity was not achieved even when a high detection accuracy of the marker treatment and setup was maintained. The problem of patient positioning on the treatment table does not represent the matching of two identical images. These images are a little bit altered because of intermarker distance variability and especially different geometry at the time of image acquiring. Patient rotation within a few degrees in a lateral direction contrary to the setup image causes a marker shift of several millimeters on the image. For this reason patient laser-tattoo alignment remains a necessary step to decrease body rotation.

The whole fraction delivery with patient positioning for prostate treatment could take about fifteen to thirty minutes. Naturally, the gland or even the whole patient performs movements over this period of time (14-16). The extent of those movements is important to know for safety margin construction. In our study, we concluded that a one centimeter margin is safe for treatment, but some extreme instances could occur.

\section{Conclusions}

Patient positioning using intraprostatic fiducial markers during radiotherapy appears to be one of the most precise methods to ensure the correct position of the target volume relative to the Clinac output. Although accuracy in patient positioning seems to allow for a significant safety margin reduction, other uncertainties enter the irradiation process. Intrafraction prostate movement in particular argues against reducing the margin below the commonly used 7-10 $\mathrm{mm}$. The results of daily marker monitoring showed that a time trend in prostate volume could be observed.

\section{Acknowledgements}

This study was supported by Charles University Grant Agency, no. 144210.

\section{References}

1. Kuban DA, et al., Long-term results of the M. D. Anderson randomized doseescalation trial for prostate cancer. Int J Radiation Oncology Biol Phys. 2008; 70 $67-74$.

2. Zietman AL, et al., Comparison of conventional-dose vs. high-dose conformal radiation therapy in clinically localized adenocarcinoma of the prostate. JAMA 2005; 294: 1233-1239.

3. Dearnaley DP, et al., Comparison of radiation side-effect of conformal and conventional radiotherapy in prostate cancer: a randomized trial. Lancet 1999; 353: 267-272.

4. Smitsmans MHP, et al., The influence of a dietary protocol on cone beam CT-guided Radiotherapy for prostate cancer patients. Int J Radiation Oncology Biol Phys. 2008; 71: 1279-1286.

5. Poulsen PR, et al., Residual set-up errors and margins in on-line image-guided prostate localization in radiotherapy. Radiotherapy and Oncology 2007; 85: 201-206.

6. Frank SJ, et al., Quantification of prostate and seminal vesicle intrefraction variation during IMRT. Int J Radiation Oncology Biol Phys. 2008; 71: 813-820. 
7. Pouliot J, et al., (Non)-migration of radiopaque markers used for on-line localization of the prostate with an electronic portal imaging device. Int J Radiation Oncology Biol Phys. 2003; 56: 862-866.

8. Kupelian PA, et al., Intraprostatic fiducials for localization of the prostate gland Monitoring intermarker distances during radiation therapy to test for marker stabil ity. Int J Radiation Oncology Biol Phys. 2005; 62: 1291-1296.

9. Sanguineti G, et al., Neoadjuvant androgen deprivation and prostate gland shrinkage during conformal radiotherapy. Radiotherapy and Oncology 2003; 66 : 151-157.

10. Zechmann CM., et al., Changes of prostate gland volume with and without androgen deprivation after intensity modulated radiotherapy. Radiotherapy and Oncology 2009; 90: 408-412.

11. Van der Heide UA., et al., Analysis of fiducial marker-based position verification in the external beam radiotherapy of patients with prostate cancer. Radiotherapy and Oncology 2007; 82: 38-45.
12. Van der Wielen GJ, et al., Deformation of prostate and seminal vesicles relative to intraprostatic fiducial markers. Int J Radiation Oncology Biol Phys. 2008; 72 1604-1611.

13. Budiharto T, et al., A semi-automated 2D/3D marker-based registration algorithm modelling prostate shrinkage during radiotherapy for prostate cancer. Radiotherapy and Oncology 2009; 90: 331-336.

14. Beltran C, Herman MG and Davis BJ, Planning Target margin calculations for prostate radiotherapy based on intrafraction and interfraction motion using four localization methods. Int J Radiation Oncology Biol Phys. 2008; 70 : 289-295.

15. Mah D, et al., Measurement of intrafractional prostate motion using magnetic resonance imaging. Int J Radiation Oncology Biol Phys. 2002; 54: 568-575.

16. Litzenberg DW, et al., Retrospective analysis of prostate cancer patients with implanted gold markers using off-line and adaptive therapy protocols. Int J Radiation Oncology Biol Phys. 2005; 63: 123-133.

Received: 24/05/2011

Accepted in revised form: 11/10/2011

\section{Corresponding author:}

Ing. Linda Kašaová, University Hospital Hradec Králové, 581 Sokolská, 50005 Hradec Králové, Czech Republic; e-mail: kasaova@fnhk.cz 